
reprints@futuremedicine.com

Controversy reigns after findings by scientists at Advanced Cell Technology Inc. indicate that stem cell lines can be derived from embryos without their destruction. Will this new technique finally settle the stem cell debate or just fan the flames of dispute?

\title{
Is embryonic destruction still an inevitable consequence of stem cell generation?
}

Recent findings by company scientists at Advanced Cell Technology Inc. (ACT) have shown that human embryonic stem cells can be derived using a novel approach that does not cause harm to the embryo.

The news that stem cell lines can be formed from embryos without destroying their potential for life has been hailed as a scientific milestone and a potential end to the deadlock surrounding funding of new embryonic stem cell research. ACT had previously reported the successful use of a

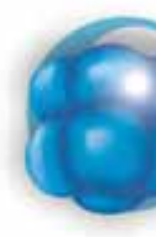
similar technique in mice in $\mathrm{N}$ ature in 0 ctober 2005 but, thus far, the technique had not been applied to human embryos.

The latest article, appearing in an online version of the journal $\mathrm{N}$ ature, is hoped to alleviate concerns surrounding the debate on embryonic stem cells, particularly from those with religious and moral objections.

Researchers, led by Robert Lanza M D, $V$ ice President of $R \& D$, outlined the technique used in their methods; it is more notably used in in vitro fertilization (IVF) clinics to assess the genetic health of preimplantation embryos. Their methods used a single-cell biopsy technique of deriving stem cells from human blastomeres, known as preimplantation genetic diagnosis (PGD).

Presently, human embryonic stem (hES) cells are derived from the inner cell mass of blastocysts, which destroys the embryo's development potential. Using the PGD technique, Lanza and colleagues extracted single cells from 16 embryos (all of which had been produced by IVF for clinical reasons) at the eight-cell stage, later developing new ES cell lines out of two of those cells.

Remarkably, the cell lines produced appear to be identical to $\mathrm{hES}$ cell lines derived from later-stage embryos using current techniques that result in the destruction of ex vivo embryos. The researchers reported that thehES cell lines maintained undifferentiated proliferation for morethan 8 months and showed normal karyotype and expression of markers of pluripotency, including $0 \mathrm{ct}-4$, SSEA-3, SSEA-4, TRA-1-60, TRA-1-81, nanog and

alkalinephosphatase. Crucially, thesecells retained the potential to form derivatives of all three embryonic germ layers both in vitro and in teratomas, thereby exhibiting their potential use in regenerative medicine.

By growing the single blastomere overnight, the resulting cells could be used for both

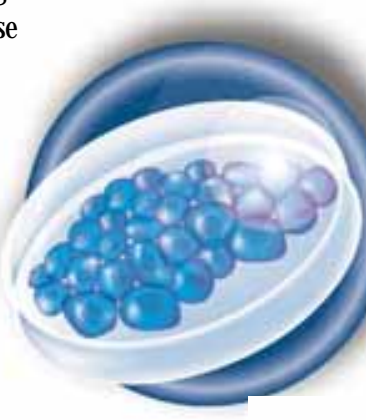
genetic testing and stem cell derivation without affecting the clinical outcome of the procedure.

"O ne of the major ethical objections of those who oppose the generation of human embryonic stems cells is that all techniques, until now, have resulted in the destruction of the embryo," stated Ronald Green, D irector of D artmouth College's Ethics Institute ( $\mathrm{N} \mathrm{H}$, U SA) and Chairman of ACT'sEthics Advisory Board. "T his technique overcomes this hurdle and has the potential to play a critical role in the advancement of regenerative medicine. It also appears to be a way out of the current political impasse in this country and elsewhere."

"U ntil now, embryonic stem cell research has been synonymous with the destruction of human embryos," stated Lanza. "I think this changes that paradigm."
D espite the initial positive reaction to the published study, a more careful examination of the evidence has revealed that the study's findings are perhaps not as clear cut as it would first appear. Several groups are now drawing attention to the fact that the research required a total of 91 individual cells to create the cell lines, resulting in multiplecellsbeing pulled from each of the 16 embryos, thereby resulting in their destruction.

"So many things about the paper and how it was presented are unclear," declared $H$ ans Schöler, a director of the $M$ ax Planck

Institute for M olecular Biomedicine in

M ünster, Germany. Schöler was also said to be disappointed that such confusion could arise in the field of stem cell technology so soon after the W oo Suk H wang scandal, when the South Korean Scientist

fraudulently claimed to have created embryonic stem cell lines from cloned human embryos.

Although Lanza's article only proposes this method, it does demonstrate that it is theoretically possible to grow ES cell lines and still

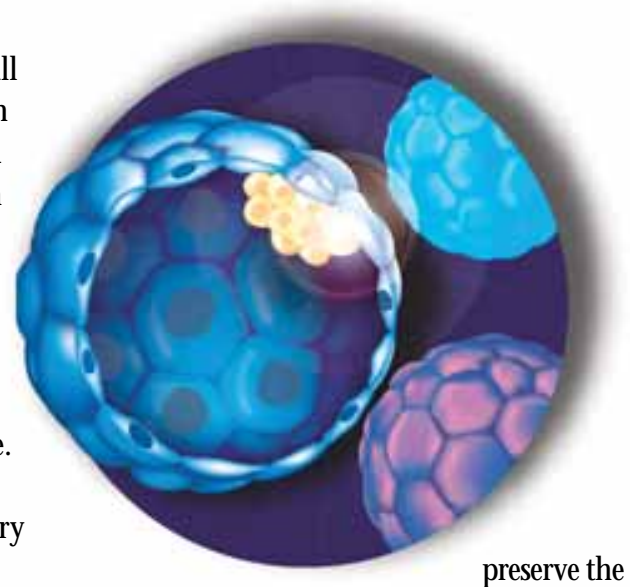

embryos. It ishoped that future experiments will further this research and essentially help overcome the continuous ethical problems facing the field. 


\section{Unravelling the stem cell differentiation enigma}

Experimental work by researchers at the University of Chicago (IL, USA) has led to the first mathematical model of a regulatory circuit used by hematopoietic stem cells to decide which type of white blood cell they will become. According to the researchers, the model is said to explain the puzzling behavior of differentiating stem cells, in which they simultaneously assume genetic signatures of different cell types before committing to one identity.

The new model, designed to understand the circuitry that controls blood cell fate determination is hoped to provide a further step towards the induction of immature stem cells to develop into specific cell types for therapeutic purposes.

The researchers, led by $\mathrm{H}$ oward $\mathrm{H}$ ughes M edical Institute investigator $\mathrm{H}$ arinder Singh, published their findings in the August 25, 2006, issue of the journal Cell. Although the studies were conducted with hematopoietic stem cells, the researchers believe that the basic principles of the regulatory network are also likely to govern cell type determination in other tissues, such as the brain and intestine. The findings could also offer insight into leukemias, in which 'indecision' in the regulatory circuitry appears to drive cancerous proliferation.

Singh's research focused on two types of white blood cells: macrophages and neutrophils. $M$ acrophages and neutrophils differ primarily in their role in fighting infection; each cell type relying on its own set of functionally active genes to carry out its particular function. H owever, before reaching maturity, both cell types and the genes that they express are identical. The primary aim of the research was to determine the processes by which a myeloid progenitor cell becomes either a macrophage or a neutrophil.

Previously, the molecular mechanisms underlying the patterns exhibited during cell fate determination have not been explored due to a lack of an experimental cell system that researchers could manipulate. Singh and colleagues used findings from their lab and others to develop such a system. Earlier studies identified transcription factors PU . 1 and C/EBPa as central genetic switches, which act to trigger myeloid progenitor cell development.

'The new model is hoped to provide a further step towards the ind uction of immature stem cells to develop into specific cell types for therapeutic purposes.'

Both factors triggered development, but crucially, how they interacted to govern stem cell differentiation remained unknown. "Both macrophages and neutrophils have very high levels of PU .1 and C/EBPa," Singh stated. "T hey both appear to directly activate a mix of both macrophage- and neutrophilspecific genes. Yet in macrophages, the neutrophil genes are off, and in neutrophils, the macrophage genes are off...So the conundrum is how a shared set of primary regulators acting on these macrophage and neutrophil genes could nevertheless produce a specific pattern of gene expression in each of the two cell types. In other words, why don't PU .1 and C/EBPa inappropriately activate neutrophil genes in the context of a macrophage, and vice versa?"

The research team used mouse progenitor cells lacking PU 1 to explore this question, manipulating the cells' decision-making machinery by altering the levels of PU.1. When low concentrations of PU .1 were introduced, the cells activated macrophage and neutrophil genes; whilst increased PU 11 levels were found to promote macrophage differentiation. Initially, the cells went through a transitory mixed lineage state, but then activated new regulatory proteins that repressed neutrophil genes and activated macrophage genes. A counteracting repressor protein was also identified that actively shuts off macrophage favoring genes during neutrophil differentiation.

It was from these and other experiments with genetically altered mouse progenitor cells that Singh and colleagues formulated their mathematical model depicting the regulatory network governing progenitor cell development. "O ur network has the property that when there are low levels of the primary regulators - $\mathrm{PU} .1$ or $\mathrm{C} / \mathrm{EBPa}$ - there is a mixed lineage pattern of gene expression," said Singh. "As we arbitrarily increase one of the two regulators, we have built into the model the counteracting regulatory circuitry that causes the genetic pattern to begin to resolve in one developmental direction or the other."

The researchers claim that the model "exhibits both graded and bistable behaviors and accounts for the onset and resolution of mixed lineage patterns during cell fate determination" and could help explain how cells in many tissues or organs can develop either via instructive regulatory signals or via noninstructive or random regulatory programs in which the cells become 'bistable' before developing into one cell type or another at random.

Such insights could have important implications for the clinical use of stem cells to rejuvenate tissues damaged by disease or trauma, stated Singh. "T here is a lot of excitement in stem cell biology these days about the possibility of rationally and efficiently generating particular cell types from different stem cells for therapeutic purposes," he said. "As we better understand the underlying genetic circuitry that orchestrates development of a particular kind of stem cell into a specialized cell type, we should be able to manipulate it for such purposes." 


\section{Imitation is the cellular form of flattery}

$\mathrm{N}$ ew research has revealed that adult stem cells are strongly influenced by the 'feel' of their surroundings as they develop into bone, muscle, neurons or other types of tissue. Researchers from the U niversity of Pennsylvania (USA) have found that physical clues from the cell microenvironment may ultimately decide which type of tissue a stem cell becomes.

The researchers were ableto manipulate naive mesenchymal stem cells by adjusting the lasticity of the gel on which they were cultured. They found that soft gels that mimic conditions in the brain produce neurogenic cells, whereas stiffer matrices, similar to muscle, gave myogenic cells and rigid microenvironments guided the cells towards becoming bone. "Basically, mesenchymal stem cells feel where they're at and become what they feel," said D ennis Discher, a Professor in Penn's School of Engineering and Applied Science. "The results begin to establish a physical basis for both stem cell use against diseases and for stem cell behavior in embryonic development."

Thefindings, published in theAugust 25 issue of the journal Cell, are expected to transform the way in which people work with stem cells. To date, the majority of research has focused on the chemical conditions governing stem cell differentiation. This is the first study to demonstrate that the physical microenvironment is also highly influential in the maturation process. "W hile I anticipated that the physical environment might limit the fate of stem cells, I never really thought that it would be sufficient to direct cell fate," said Lee Sweeney, chairman of Physiology in Penn's School of M edicine. "I was stunned to see that the physical environment alonewastelling the stem cells to become neurons or muscle or bone."

\section{H owever, physical} microenvironments can change as a result of injury or disease, which could make it difficult to use these stem cells in certain types of therapy. For example, the scarred tissue that remains after a heart attack could prevent stem cells from developing into cardiac muscle. As D ischer explains, "The cardiac tissue may have been so damaged during the heart attack that the stem cells do not recognize the microenvironment as a guide for turning into heart muscle."

$\mathrm{N}$ evertheless, Discher is confident that the findings could lead to breakthroughs in the treatment of many diseases. "O ur studies show that it might be possible to 'prime' stem cells for therapy in the lab, before implanting them in the heart, spine or whatever damaged environment you want to place them".

\section{D iabetes cure for rats}

Researchers from the Washington University School of M edicine in St Louis (M O, USA) have reported that a technique al ready proven to cure a rat model of Type 1 or juvenile-onset diabetes can al so be used to successfully treat Type 2 or adult-onset diabetes. Their results are published in Transplant I mmunology.

In a previous study, senior author $\mathrm{M}$ arc $\mathrm{H}$ ammerman and coauthor Sharon Rogers demonstrated that they could transplant pancreatic primordial from pigs into rats and allow them to develop into insulin producers without being rejected by the immune system.

Consequently, as well as curing the rats' diabetes, the use of immune suppression drugs - which can sometimes be hazardous - was avoided. As part of this investigation, the team transplanted pig pancreatic primordia into groups of rats with a disorder similar to that of Type 2 diabetes found in humans. In a similar outcome to their earlier study, the rats' diabetes was cured without the need for immune suppression drugs.

$\mathrm{H}$ ammerman explained the results of the study: "The transplanted primordia not only appropriately regulated blood sugar in the Type 2 diabetic rats, they also reduced insulin resistance. The rats are cured by pig insulin, which comes from the transplants and can be measured in their circulation. The rats' own insulin-producing cells in the pancreas are atrophied."

The findings al so confirm that the positive immune response observed from the first study was not owing to the strain of rats used; "In addition, now we also know that this approach works for the much more common Type 2 diabetes, something we couldn't predict based on our earlier research."
The results of the study have potentially significant implications on the treatment of diabetes. W hilst injection of insulin may not be sufficient to prevent diabetic complications, transplanted insulinsecreting cells will function only during periods of high blood sugar levels and will stop producing the hormone once normal glucose levels have been reached.

The researchers have begun to expand their study by transplanting pig pancreatic primordia into diabetic primates. If proven succesfful, trials in humans will be the next stage for the investigators and, as described by $\mathrm{H}$ ammerman, may have important consequences on diabetes therapy: "If pig pancreatic primordia prove to be comparably invisible to the human immune system, they could represent a virtually unlimited source of donor organs to treat human diabetes without the need for host immune suppression." 


\section{in brief. .}

CD26 inhibition enhances allogeneic donor cell homing and engraftment after in utero hematopoietic cell transplantation.

Peranteau WH, Endo M, Adibe 00, Merchant A, Zoltick PW, Flake AW: Blood (2006) [Epub ahead of print].

The authors hypothesized that CD26 inhibition would increase donor cell homing to the fetal liver and improve allogeneic engraftment following in utero hematopoietic cell transplantation (IUHCT). B6GFP bone marrow (BM) or enriched hematopoietic stem cells (HSCs) were thus transplanted into allogeneic fetal mice with or without CD26 inhibition. The study found that CD26 inhibition resulted in: increased allogeneic BM and HSC homing to the fetal liver; postnatal engraftment evident in increased animal numbers; increased donor chimerism levels following IUHCT; competitive engraftment advantage over noninhibited congenic donor cells. CD26 inhibition could thus be a potential method for increasing the level of fetal liver homing and engraftment following IUHCT and could be used to increase donor-specific tolerance following IUHCT in the future.

Neural stem cell properties of Muller glia in the mammalian retina: regulation by Notch and Wnt signaling.

Das AV, M allya KB, Zhao X et al.: Dev. Biol. (2006) [Epub ahead of print].

This study identifies Muller cells as latent neural stem cells (NSCs) in the mammalian retina. The researchers show that, when retrospectively enriched from the normal retina, M uller cells display cardinal features of NSC s (self-renewal and generation of all three basic cell types in the CNS). These cells also possess the potential to generate retinal neurons (in vitro and in vivo). The NSC nature of $M$ uller cells remains dormant under the constraint of the non-neurogenic environment of the adult normal retina, how ever, Muller cells can generate potential retinal neurons if activated to do so. This study determined that the Wnt and Notch signaling pathways are involved in this activation. These results suggest that Muller cells could act as a potential target for cellular manipulation in the treatment of retinal degeneration.

\section{Compounds that stimulate cell growth in the brain}

The identification of several key compounds that stimulate stem cell growth in the brain may have important implications for people suffering from neurological disorders, including Parkinson's disease. The findings, from a study published in the September issue of the FASEB Joumal, may provide more insights into what compounds cause neurons to regenerate and re-establish brain function.

The two compounds studied in particular were LT B4 and LXA4, both of which have a role in inflammation and regulate the propagation of several types of cells. In the investigation, stem cells were isolated from the brains of mouse embryos. These cells were then exposed to LT B4 and subsequently proliferated and differentiated, resulting in the production of new stem cells and neurons that were unable to or had a limited ability to divide. After exposure to LXA4, the cells demonstrated decreased growth and apoptosis.
In addition, insights into the cellular and molecular pathways involved when LT B4 stimulates neuronal stem cells were also gained from the investigation. It was found that cells produced through exposure to LT B4 had higher levels of LT B 4 receptors compared with cells that were not exposed. Furthermore, several molecules, including cyclins and epidermal growth factor receptors, involved in the cell cycle and growth were upregulated by LT B4. Similarly, molecules that have a role in apoptosis, for example, caspase 8, were decreased in the presence of LT B 4. H owever, LXA4 compounds demonstrated the opposite effect in the same cells.

The significance of the study was highlighted by G erald Weissmann, Editor-in-C hief of the FASEB Jamal: "T his study opens doors to new therapeutic approaches for a wide range of neurological disorders and injuries that were once considered incurable."

\footnotetext{
About the Bulletin Board newsworthy information, please contact:

Elisa Manzotti,

Editor, Regenerative Medicine,

Future Medicine Ltd, Unitec House,

2 Albert Place, Finchley Central, London N3 1QB, UK

E-mail: e.manzotti@futuremedicine.com

Tel: +44 (0)20 8349 2033; Fax: +44 (0)20 83432313
}

The Bulletin Board highlights some of the most important events and launches in stem cell research and regenerative medicine. The editorial team welcomes suggestions for timely, relevant items. If you have 